\title{
Nuevos modelos de docencia en Grados de Comunicación: Portales informativos digitales como herramienta de motivación
}

\author{
Rafael CARRASCO POLAINO \\ Universidad Complutense de Madrid \\ racarras@ucm.es \\ Miguel Ángel MarTín CÁRDABA \\ Centro Universitario Villanueva, Adscrito a la Universidad Complutense de Madrid \\ mmartincar@villanueva.edu
}

\begin{abstract}
Resumen
cuv3.com es un portal informativo digital en donde los alumnos de Comunicación del Centro Universitario Villanueva, adscrito a la Universidad Complutense de Madrid, publican desde los primeros cursos de forma voluntaria o, como parte práctica de algunas asignaturas contenidos profesionales multimedia. Ofrecer metodologías de este tipo puede ofrecer beneficios al menos en tres ámbitos: motivación, satisfacción y cumplimiento de competencias.
\end{abstract}

Palabras clave: Comunicación; Universidad; Motivación; Herramientas; Competencias

\section{New models of teaching in Communication Degrees: digital information media as a motivational tool.}

\begin{abstract}
cuv3.com is a digital information media where students of Communication in Villanueva University Center, affiliated with the University Complutense of Madrid, publish since the first courses a voluntarily or as part of some subjects media professional content.

These methodologies may provide benefits in at least three areas: motivation, satisfaction and fulfillment of responsibilities.
\end{abstract}

Key Words: Communication; University; Motivation; Tools; Competences

\section{Referencia normalizada:}

Carrasco Polaino, R.; M. Cárdaba, M. A. (2013) Nuevo modelos de docencia en Grados de Comunicación: Portales informativos digitales como herramienta de motivación. Historia y Comunicación Social. Vol. $18 \mathrm{~N}^{\circ}$ Especial Octubre. Págs. 421-432.

Sumario: 1.- Introducción. 2.- Participantes, método y procedimiento. 3.- Resultados. 4.- Discusión y conclusiones. 


\section{Introducción}

Desde la incorporación definitiva del Espacio Europeo de Educación Superior en España, en el año 2010 (Espacio Euopeo Educación Superior), muchas han sido las opiniones que han apoyado o criticado el nuevo sistema denominado "de Bolonia" (García Redondo, Lázaro Herrero, \& Martín Sánchez, 2010), en el que no sólo se han modificado los planes de estudios de todas las licenciaturas y diplomaturas, transformándolos en Grados o incluso haciendo que algunas diplomaturas desaparezcan, si no también las nuevas metodologías dicentes que se plantean, totalmente centradas en el protagonismo del alumno en detrimento del profesor.

La utilización de recursos de carácter tecnológico para desarrollar los nuevos sistemas metodológicos ha sido una de las tendencias más seguidas por universidades y profesores, ya sea para el trabajo en el aula o para el seguimiento y tutorización de trabajos tanto individuales como en grupo fuera de ella. (Menedez, 2010).

De cara a cumplir con los objetivos presentados por el Espacio Europeo de Educación Superior en donde una de las metodologías que se instauran es la de la enseñanza práctica a través de la intervención activa del alumno a través de ejercicios, trabajo en grupo, prácticas profesionales, etc, (Ministerio de Educación, Cultura y Deporte) el Centro Universitario Villanueva desarrolló, dentro de la asignatura Producción Periodística en Nuevas Tecnologías, de $3^{\circ}$ de la Licenciatura de Periodismo, en el año 2010, un portal informativo digital y multimedia en el que los estudiantes de las áreas de estudio de Periodismo, Publicidad y RR.PP. y Comunicación Audiovisual pudieran desarrollar esta metodología práctica.

El portal de información, cuv3.com, fue desarrollado íntegramente por los alumnos de este curso de $3^{\circ}$ de Periodismo durante el cuatrimestre que duro la asignatura anteriormente mencionada y resultó de tanto éxito en cuanto a usuarios que los profesores responsables del proyecto decidieron continuar desarrollándolo junto con los alumnos tanto dentro de la dinámica de algunas asignaturas como de forma voluntaria por parte de los alumnos.

En este portal los alumnos publican, de forma tutelada y bajo la supervisión de profesores, noticias y reportajes tanto de actualidad general como de la actualidad de la universidad además de difundir trabajos y proyectos que van realizando en las diferentes asignaturas.

La metodología de trabajo presenta varias fases:

El la primera de ella el alumno propone un tema de publicación al responsable editorial del portal. Este responsable ayuda al alumno a elegir un correcto enfoque del tema.

En una segunda fase el alumno, por su cuenta, si es una publicación voluntaria o, tutelado por un profesor si se trata de una actividad vinculada a una asignatura, elabora los contenidos informativos y los envía a la dirección técnica y redaccional. 
La corrección técnica y redaccional se realiza en una tercera etapa, en donde, si la información es correcta se publica automáticamente. Si no lo es, el alumno junto con los profesores correspondientes corrigen los posibles errores.

Una vez que el alumno corrige estos posibles errores vuelve a enviar la información a la fase de revisión, en donde se comprueba que todo es correcto y la información pasa a ser publicada.

Con este sistema se consigue que el estudiante conozca sus errores, comprenda su origen, sepa como corregirlos y, al corregirlos el mismo, el conocimiento queda adquirido y no se vuelven a repetir.

Tras tres años de utilización de esta metodología el portal no ha dejado de crecer en número de colaboradores, informaciones, usuarios e incluso ingresos generados por publicidad o por la producción de contenidos bajo demanda.

La elaboración de trabajo texto surge por la necesidad de comprobar la sensación que tienen los autores de que la posibilidad que tienen los alumnos trabajar siguiendo criterios profesionales en Cuv3, de aplicar la formación obtenida en asignaturas regladas y la posibilidad de publicar y difundir sus trabajos y proyectos universitarios presenta beneficios al estudiante en tres aspectos: la experiencia del estudiante en la vida universitaria, un mejor desarrollo de la profesión y sentimiento de reconocimiento en el trabajo y una mejor consecución de las competencias transversales y generales que se proponen en los planes de estudio de los grados de Periodismo, Publicidad y RR.PP. y Comunicación Audiovisual.

Para comprobar estas hipótesis se generó una metodología que se describe a continuación.

\section{Participantes, método y procedimiento}

Treinta y siete estudiantes del área de comunicación del Centro Universitarios Villanueva (UCM) completaron, de manera anónima y voluntaria, un cuestionario online en el que evaluaron su participación en el programa educativo Cuv3. Las preguntas de el cuestionario se agrupaban primordialmente en torno a tres áreas principales: 1) posibles beneficios de la participación en Cuv3 sobre aspectos relevantes de la vida universitaria en general (e.g., motivación, aprendizaje, mentalidad de trabajo, etc); 2) posibles beneficios de la participación en Cuv3 sobre las competencias previstas por el plan educativo Bolonia respecto a cada uno de los grados (Periodismo, Publicidad y RRPP, y Comunicación Audiovisual); y 3) posibles beneficios de la participación en Cuv3 de cara a la inserción y la experiencia en el mundo laboral y profesional. A lo largo del cuestionario, se pidió a los participantes que mostraran su opinión respecto a cada uno de los ítems completando una escala de cuatro opciones (totalmente en desacuerdo; en desacuerdo; de acuerdo; totalmente de acuerdo) 


\section{Resultados}

3.1. Beneficios de cuv3 sobre aspectos relevantes de la vida universitaria

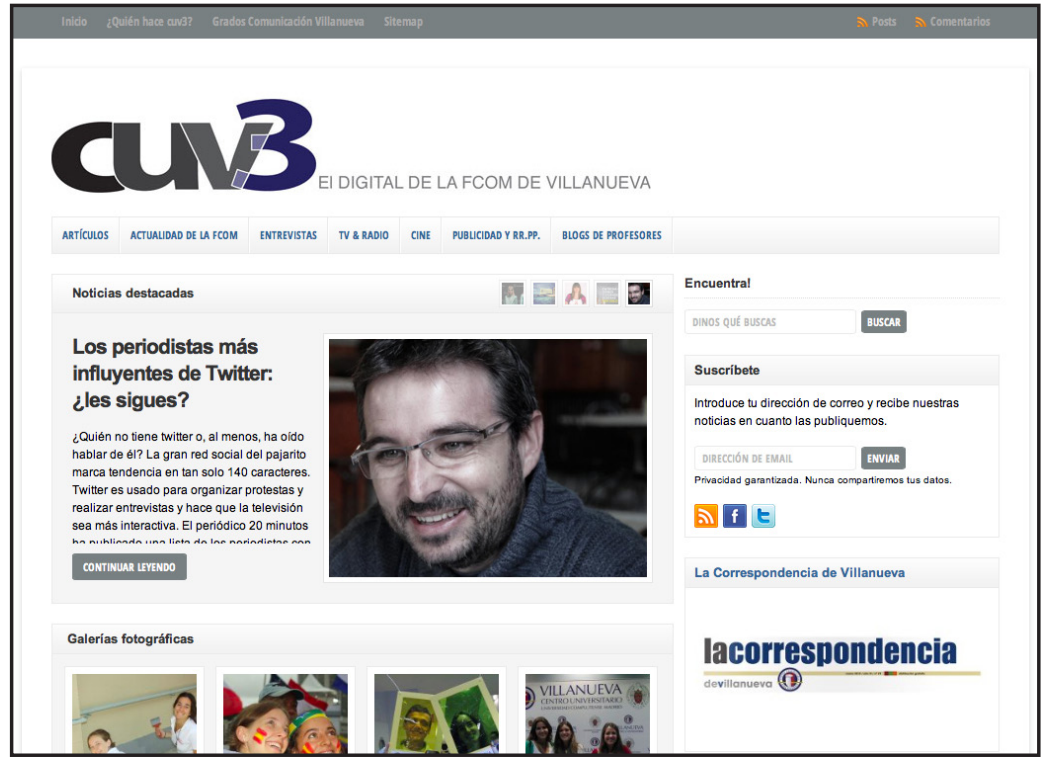

El 74\% de los estudiantes declaran estar de acuerdo o muy de acuerdo con la afirmación de que su participación en Cuv3 ha fomentado su motivación respecto a sus estudios universitarios. (Gráfico1)

\section{Gráfico 1}

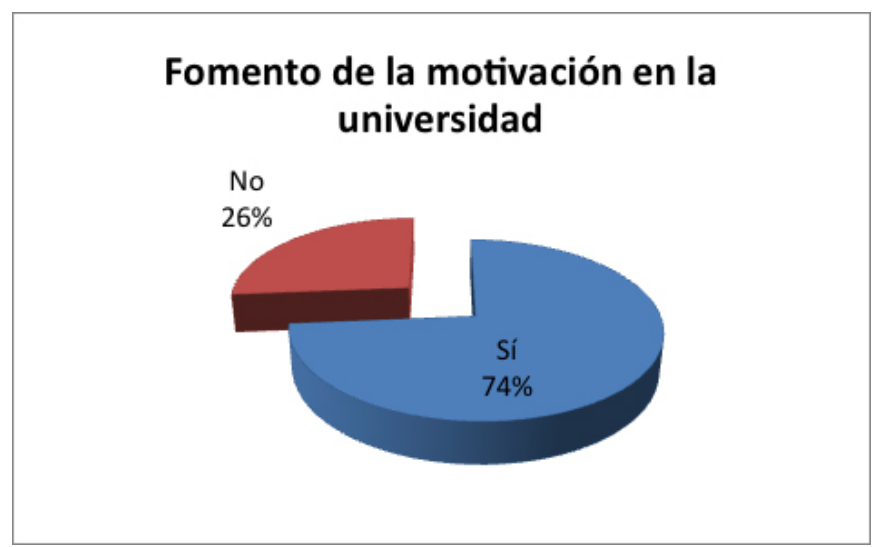

El 66\% declaran que su participación en Cuv3 les ha ayudado a vivir más y mejor el espíritu universitario. (Gráfico2) 


\section{Gráfico 2}

\section{Fomento del espíritu universitario}

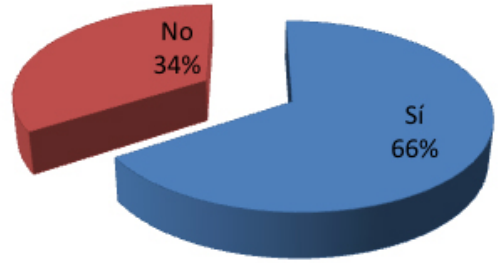

El 76\% dicen que su participación en Cuv3 les ha ayudado a mejorar su proceso de aprendizaje general. (Gráfico3)

\section{Gráfico 3}

\section{Mejora el proceso general de aprendizaje}

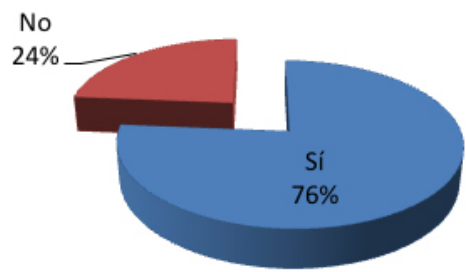

El 39\% reconoce que su participación en Cuv3 ha colaborado para mejorar sus resultados académicos. (Gráfico4)

\section{Gráfico 4}

\section{Mejora resultados académicos}

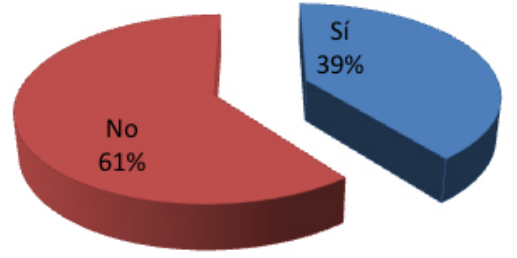


El 71\% reconoce que su participación en Cuv3 ha hecho que disfruten más de su experiencia universitaria. (Gráfico5)

\section{Gráfico 5}

\section{Fomenta el disfrute de la experiencia universitaria}

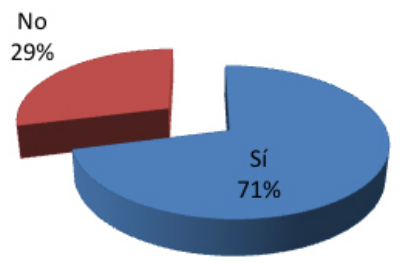

El 47\% afirma que su participación en Cuv3 les ha ayudado a recordar mejor lo aprendido en las asignaturas de sus estudios. (Gráfico6)

\section{Gráfico 6}

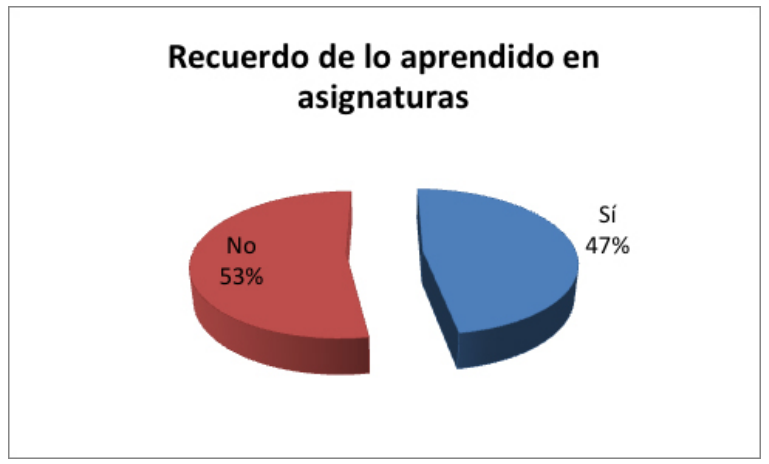

El 87\% declara que su participación en Cuv3 les ha ayudado a desarrollar una mentalidad más profesional. (Gráfico7)

\section{Gráfico 7}

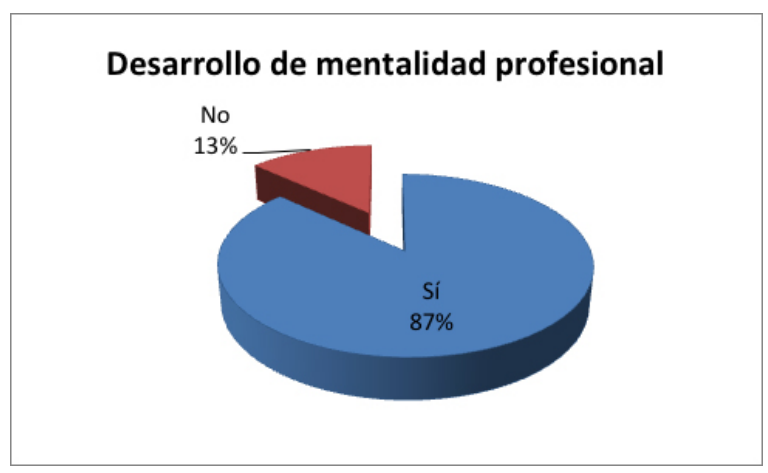


3.2.- Beneficios de cuv3 respecto a la las competencias tranversales y generales correspondientes a cada grado

El 76\% (28)son alumnos de periodismo, el 14\% (5) de publicidad y relaciones públicas y el 11\% (4) de comunicación audiovisual

\section{Periodismo}

El $82 \%$ de los estudiantes de periodismo reconoce estar de acuerdo o muy de acuerdo con la afirmación de que su colaboración en Cuv3 ha mejorado su competencia para "utilizar correctamente el lenguaje oral y escrito y capacidad y habilidad para expresarse con fluidez y eficacia comunicativa en la comunicación verbal y no verbal".

El 82\% declara que su colaboración en Cuv3 ha aumentado su "capacidad para buscar, seleccionar, interpretar y analizar textos y documentos".

El 89\% declara que su colaboración en Cuv3 ha incrementado su "iniciativa, creatividad credibilidad, honestidad, imagen, espíritu de liderazgo y responsabilidad tanto de modo individual como corporativo".

E1 79\% afirma que su colaboración en Cuv3 ha mejorado su "capacidad para explicar y relacionar conocimientos".

El 75\% asevera que su colaboración en Cuv3 ha aumentado su "capacidad para experimentar e innovar mediante el conocimiento y uso de métodos y técnicas aplicados".

El 68\% afirma que su colaboración en Cuv3 ha mejorado su "conocimiento de los aspectos sociales, económicos y culturales del contexto nacional e internacional y las estructuras, características y configuraciones de las sociedades".

El 68\% reconoce que su colaboración en Cuv3 ha aumentado su "conocimiento, defensa y difusión de los derechos humanos y garantías constitucionales en las informaciones periodísticas y divulgativas que lo exijan".

El 64\% declara que su colaboración en Cuv3 ha incrementado su "capacidad para saber ejercer, en su caso, las garantías constitucionales que reconocen las libertades de empresa, de información y de expresión diferenciando los productos informativos de otros productos meramente comunicativos".

\section{Publicidad y RRPP}

El $80 \%$ de los estudiantes del Grado de Publicidad y RRPP reconoce estar de acuerdo o muy de acuerdo con la afirmación de que su colaboración en Cuv3 ha contribuido a mejorar su "capacidad para obtener provecho de las diversas fuentes de información al alcance del alumno en las distintas materias de estudio para maximizar su formación y alcanzar así un rendimiento óptimo en el plano académico, personal y, posteriormente, profesional". 
El 100\% declara que su colaboración en Cuv3 ha incrementado su "capacidad para desarrollar un razonamiento crítico coadyuvante en cualquiera de los contextos que van a conformar su futuro profesional".

El 100\% afirma que su colaboración en Cuv3 ha aumentado su "capacidad para la detección, el análisis y la resolución de problemas en cualquiera de los ámbitos que van a conformar la vida académica y profesional del alumno; y para la aplicación de métodos instrumentales de carácter interdisciplinar necesarios para ello".

El 100\% reconoce que su colaboración en Cuv3 ha acrecentado su "habilidad para poner en práctica un comportamiento creativo en aquellas situaciones que requieran de una solución novedosa y eficaz; involucrándose en su ejecución y adoptando un funcionamiento ágil y flexible".

El 100\% asevera que su colaboración en Cuv3 ha incrementado su "capacidad para el trabajo e interactuación en equipos multi e interdisciplinares, contribuyendo a la consecución de los objetivos previamente establecidos, y revirtiendo sus resultados en el aprendizaje personal y colectivo".

El 100\% declara que su colaboración en Cuv3 ha favorecido su "capacidad para adquirir un compromiso ético a nivel personal y social, manifiesto tanto en su comportamiento académico, como en su futuro quehacer profesional".

\section{Comunicación Audiovisual}

El 100\% de los estudiantes del Grado de Comunicación Audiovisual reconoce estar de acuerdo o muy de acuerdo con la afirmación de que su colaboración en Cuv3 ha contribuido a mejorar su "conocimiento de las bases expresivas del lenguaje. Abarcando todo lo relativo a la comunicación, lenguaje y habla.

El 100\% declara que su colaboración en Cuv3 ha favorecido su "conocimiento de los mecanismos psicológicos y sociales que explican el origen, desarrollo y cambio de la comunicación audiovisual".

El 75\% afirma que su colaboración en Cuv3 ha incrementado su "conocimiento de los fundamentos artísticos, históricos, literarios, sociológicos, económicos, etc., que le permitan decidir mediante criterios propios y hacerse aconsejar ante cada producción audiovisual".

El 50\% declara que su colaboración en Cuv3 ha aumentado sus "conocimientos para aplicar técnicas de evaluación de los resultados de la programación audiovisual y sus efectos sociales y económicos".

El 100\% reconoce que su colaboración en Cuv3 ha favorecido su "conocimiento para buscar, seleccionar y sistematizar cualquier tipo de documento audiovisual en una base de datos, así como su utilización en diferentes soportes audiovisuales utilizando métodos digitales"

El 25\% asevera que su colaboración en Cuv3 ha favorecido su "conocimiento del ordenamiento jurídico, de la ética y deontología que regulan la producción audiovi- 
sual que incluye los derechos fundamentales implicados en la comunicación audiovisual y la libertad de comunicación. En concreto sobre la normativa audiovisual, derechos de autor, jurisprudencia y doctrina jurídica audiovisual”.

3.3.- Beneficios de cuv3 respecto al desempeño en el mundo profesional

El 73\% de los estudiantes que han colaborado con Cuv3 han tenido, a su vez, experiencia profesional posterior a su participación en Cuv3. De entre todos ellos:

E1 93\% afirma estar de acuerdo o muy de acuerdo en que, tras su participación en Cuv3, se han sentido más preparados para el desempeño de sus tareas profesionales. (Gráfico8)

\section{Gráfico 8}

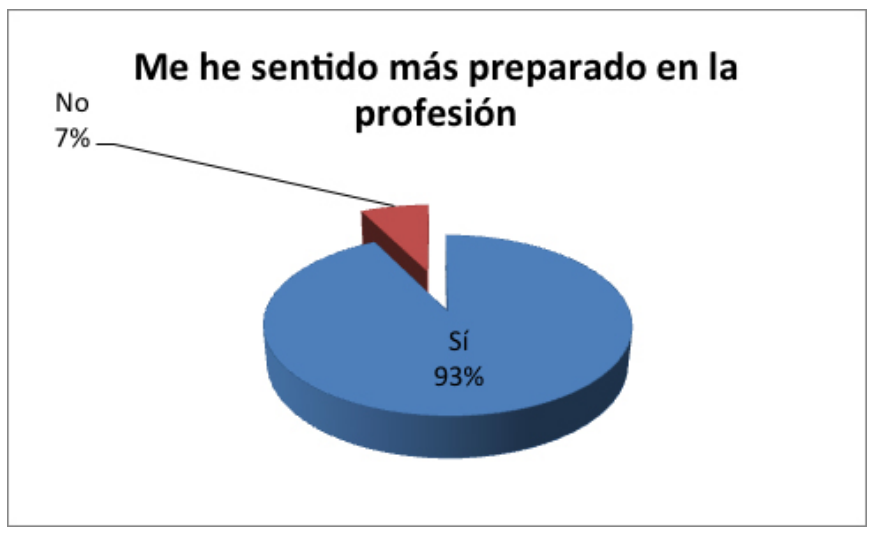

El $85 \%$ declara haber sentido mayor confianza a la hora de llevar a cabo tareas encomendadas. (Gráfico9)

\section{Gráfico 9}

\section{Mayor confianza en el trabajo}

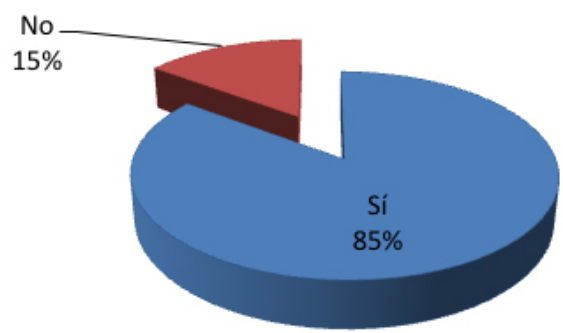


El 63\% asevera haber sentido mayor reconocimiento por parte de sus superiores. (Gráfico10)

Gráfico 10

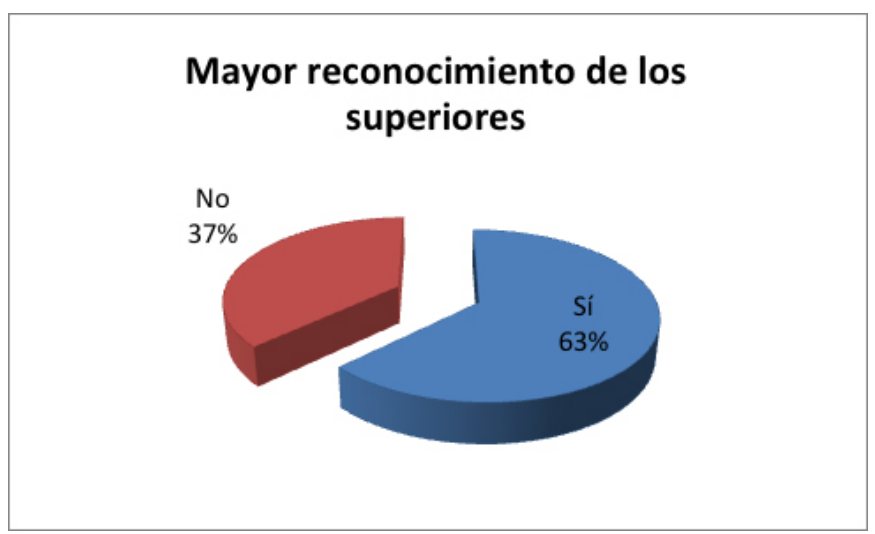

El 67\% afirma que su participación en Cuv3 les ha ayudado a disfrutar más en el trabajo. (Gráfico11)

\section{Gráfico 11}

\section{Mayor disfrute en el trabajo}

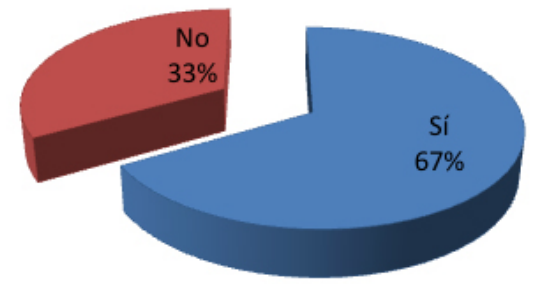

Por último, como quedó anteriormente establecido, los estudiantes que participaron en Cuv3 lo hicieron o bien como parte de una asignatura o bien de manera voluntaria (el 76\%). Resulta relevante destacar que, de todos los que eligieron participar de manera voluntaria en el programa, el 100\% afirmo que, de tener de nuevo la oportunidad, volvería a participar en Cuv3. 


\section{Discusión y conclusiones}

Puede concluirse que la gran mayoría de los estudiantes que han participado en Cuv3 siente que la publicación y difusión de informaciones y trabajos de forma tutelada por sus profesores consigue que los conocimientos adquiridos en las asignaturas se reflejen rápidamente en un trabajo real, lo que hace que el alumno compruebe la importancia que tiene la adquisición de conocimientos teóricos para poder desarrollar correctamente un trabajo práctico.

Que la participación en Cuv3 haya sido voluntaria o como parte de una asignatura no ha resultado de relevancia para comprobar que esta metodología resulta positiva en todos sus aspectos.

Además, se puede comprobar que las nuevas formas docentes de este perfil recomendadas por las metodologías del Espacio Europeo de Educación Superior colaboran de forma muy adecuada a que los estudiantes consigan de forma exitosa cumplir con las competencias transversales y generales correspondientes a sus Grados universitarios.

Limitaciones y futuras investigaciones: aun cuando los resultados encontrados son sugerentes e informativos, el diseño del presente estudio sólo nos permite medir las percepciones subjetivas que los estudiantes tienen sobre la adquisición de competencias y habilidades. No obstante, en futuras investigaciones, sería deseable poder comprobar, mediante un diseño experimental, la eficacia de Cuv3 en la adquisición real de dichas competencias y habilidades.

\section{Referencias bibliográficas}

GARCÍA REDONDO, E., LÁZARO HERRERO, L., \& MARTÍN SÁNCHEZ, S. (2010). El alumno iun pilar básico en el EEES? Idas y venidas en su viaje a Bolonia. En L. V. Gil, El proceso de Bolonia y la Educación Comparada. Miradas críticas (págs. 108-124). Salamanca: Ediciones Universidad de Salamanca.

MENEDEZ, F. E. (2010). Aportaciones de los entornos virtuales a la metodología del EEES. En L. V. Gil, El proceso de Bolonia y la Educación comparada. Miradas críticas (págs. 44-52). Salamanca: Ediciones Universidad de Salamanca.

\section{Notas}

Espacio Euopeo Educación Superior. (s.f.). Desarrollo cronológico. Recuperado el 30 de 09 de 2013, de Espacio Euopeo Educación Superior: http://www.eees.es/es/eees.

Ministerio de Educación, Cultura y Deporte. (s.f.). De las lecciones en clase al aprendizaje activo. Recuperado el 27 de 09 de 2013, de Qué es Bolonia: http://www.queesbolonia.gob.es/queesbolonia/ bolonia-para-ti/profesor/de-las-lecciones-en-clase-al-aprendizaje-activo.html 


\section{Los autores}

Rafael Carrasco Polaino es Doctor y licenciado en Periodismo por la Universidad Complutense de Madrid y Técnico en Diseño y Comunicación por el Instituto Europeo de Comunicación y Marketing. Acreditado por la Agencia de Calidad, Acreditación y Prospectiva de las Universidades de Madrid (ACAP) como Profesor colaborador, Profesor ayudante Doctor, Profesor Doctor de universidad privada y Profesor contratad doctor. Profesor agregado de asignaturas relacionadas con la Tecnología de la Información y la Empresa Informativa desde 1999 en Centros como la Universidad Complutense de Madrid, Centro Universitario Villanueva, Universidad Europea de Madrid (UEM) y ESIC Business and marketing School. Profesor del Master de Periodismo de ABC. Miembro del Consejo de Dirección de la revista Cuenta y Razón. ISSN 0211-1381.

Miguel Ángel M. Cárdaba es Licenciado en Filosofía por la Universidad de Navarra, Diplomado en Sociología por la Universidad Nacional de Educación a distancia y Doctor Europeo en Ciencias de la Información por el departamento de sociología VI de la UCM. Actualmente imparte clases de Psicología aplicada a la Publicidad y a las RRPP, así como de Antropología y Sociología de la Moda en el Centro Universitario Villanueva. Además es profesor de la asignatura de Comunicación y persuasión que se imparte en el Máster en Comunicación Institucional y Política que organiza la Universidad Carlos III. Autor de varios artículos en publicaciones nacionales e internacionales como Comunicar, Psicothema o el Journal of Applied Social Psychology, su investigación actual se centra en el ámbito de la Psicología social de la Comunicación. 